\title{
Emprego da Técnica de Geração e Desaparecimento de Raios na Simulação de Canal UWB em ambiente indoor
}

\author{
Rodrigo V. S. de Almeida e Leni J. de Matos
}

\begin{abstract}
Resumo - Baseada no espalhamento temporal segundo um processo de Poisson, e partindo de um modelo de propagação para a análise do efeito do desvanecimento multipercurso, no problema perto-longe em sistema rádio móvel CDMA, a técnica de Geração e Desaparecimento de Raios é aqui empregada na simulação de canal UWB indoor. Para isso, parâmetros como espalhamento de retardo, número médio de multipercursos (ou raios), tempo médio de vida de um raio, retardo médio e potência média recebida, obtidos por processamento de dados coletados de medições de sinal UWB em ambientes indoor, servem como entrada para o processo de simulação. $O$ desempenho da técnica é avaliado através da comparação do parâmetro espalhamento de retardo do canal sondado e do simulado.
\end{abstract}

Palavras-chave - Geração e desaparecimento de raios, espalhamento de retardo, canal rádio UWB

Abstract - Based on time scattering according to a Poisson process, and starting from a propagation model for the analysis of the effect of the multipath fading in the near-far problem in CDMA mobile radio system, the technique of Generation and Disappearance of Paths is here employed in the indoor UWB channel simulation. For this, parameters like delay spread, average number of paths, average life of a path, average delay and average received power, all obtained by processing of acquired data from measurements of UWB signal in indoor environments, are used as input parameters for the simulation process. The performance of the technique is valued through the comparison of the delay spread in the sounding channel and the simulated channel.

Keywords - Generation and disappearance of scattered waves, delay spread, UWB radio channel

\section{INTRODUÇÃO}

Um canal UWB (Ultra Wide-Band) possui, por definição, uma largura de banda superior a $500 \mathrm{MHz}$, conseqüentemente, permitindo maior capacidade de transmissão, e opera com uma baixa relação sinal/ruído. Uma das aplicações mais promissoras para a tecnologia UWB é a conexão sem fio de diversos equipamentos eletrônicos. Também já se vislumbra sua aplicação no resgate de vítimas sob escombros ou neve [1].

Rodrigo Vieitas Sarruf de Almeida e Leni Joaquim de Matos (orientadora), Dep ${ }^{\text {to }}$ de Engenharia de Telecomunicações, Escola de Engenharia, Universidade Federal Fluminense, Niterói, RJ, E-mails: rodrigovieitas@hotmail.com, lenijm@ig.com.br
Tal tecnologia permite taxas de transferência que podem variar de $100 \mathrm{kbps}$, para um mouse sem fio, a valores como $100 \mathrm{Mbps}$, para o download de imagens e compartilhamento de arquivos de multimídia. Tais taxas de transmissão, permitidas no canal UWB, estão relacionadas com o parâmetro de dispersão temporal do canal, conhecido como espalhamento de retardo [2], que é usado como referência, neste artigo, para avaliar a técnica de simulação empregada.

Os modelos de simulação GWSS-US (Gaussian Wide Sense Stationary - Uncorrelated Scattering) são os mais utilizados [3]. Entretanto, a utilização desses modelos possui limitações, principalmente para aplicação em sistemas de banda-larga, já que os valores de retardo são considerados invariantes no tempo, portanto, fixos. Assim, foram desenvolvidos modelos mais complexos para simulação banda larga, denominados por alguns autores de "modelos físicos" [3].

Os modelos físicos tiveram início com Turin [4], que realizou uma grande campanha de medidas envolvendo a transmissão simultânea, partindo de um ponto fixo, de pulsos de 100 ns nas freqüências de 488, 1280 e 2920 $\mathrm{MHz}$, chegando a um receptor móvel. O meio de propagação foi modelado como um filtro linear da forma:

$$
h(t)=\sum_{k=0}^{\infty} a_{k} \delta\left(t-t_{k}\right) e^{i \theta_{k}}
$$

onde $a_{k}, t_{k}$ e $\theta_{k}$ são, respectivamente, um conjunto de amplitudes, retardos e fases. Assim, este é um modelo banda-larga que, por sua generalidade, pode ser usado para obter a resposta do canal para qualquer sinal de entrada, $\mathrm{s}(\mathrm{t})$, através da convolução de $\mathrm{s}(\mathrm{t}) \operatorname{com} \mathrm{h}(\mathrm{t})$. De acordo com este modelo, mostrado na Figura 1 , o meio de propagação pode ser caracterizado por um conjunto de amplitudes, tempos de retardo e fases segundo o filtro linear e acrescido do ruído aditivo $\eta(\mathrm{t})$.

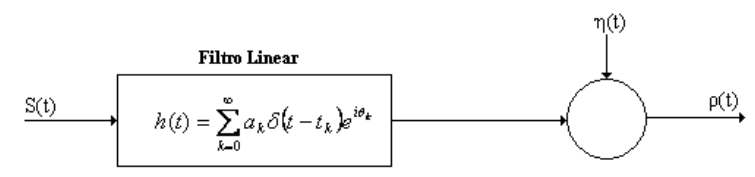

Fig. 1. Modelo Matemático do Canal para Simulação 
Posteriormente, os dados experimentais de Turin foram analisados por Suzuki [5]. Após aplicar as distribuições lognormal, Rice, Rayleigh e Nakagami, Suzuki verificou que as distribuições que melhor se ajustavam aos dados experimentais das amplitudes dos multipercursos eram Nakagami e lognormal. Para os retardos foi proposto um modelo de Poisson modificado, denominado modelo $\Delta-\mathrm{K}$.

O modelo apresentado por Turin e Suzuki foi refinado por Hashemi [6]. Para a simulação dos retardos, Hashemi substituiu o fator K de Suzuki por novos parâmetros K' que são funções do "número de bins" i (bin é um intervalo de tempo $(\Delta)$ fixo onde existe, no máximo, um multipercurso). A modificação permite que a simulação funcione para qualquer número de bins. Também foi levada em conta a correlação espacial entre os retardos e entre as fases.

Baseado nas premissas dos modelos físicos, que é caracterizar estatisticamente os parâmetros do canal, e visando analisar o canal CDMA, Iwai e Karasawa [7] desenvolveram um novo modelo de propagação banda larga através do emprego da Técnica de Geração e Desaparecimento de Raios.

O objetivo deste trabalho é aplicar esta técnica na simulação de canais UWB indoor, de faixa igual a 750 $\mathrm{MHz}$, e avaliar o seu desempenho, com base no espalhamento de retardo do canal real medido e do simulado.

A seção II deste artigo descreve a técnica desenvolvida aqui adotada, enquanto a seção III apresenta sua aplicação ao canal UWB sondado, descrevendo o ambiente de medições, especificações da técnica de sondagem empregada e os parâmetros médios obtidos experimentalmente. A seção IV apresenta os resultados das simulações com o emprego da técnica de Geração e Desaparecimento de Raios para canais UWB e a comparação entre os parâmetros simulados e obtidos experimentalmente em ambientes da Escola de Engenharia da Universidade Federal Fluminense. Finalmente, na seção $\mathrm{V}$, são apresentadas as conclusões.

\section{TÉCNICA DE GERAÇÃO E DESAPARECIMENTO DE RAIOS}

Proposta para a modelagem de canais banda larga, a Técnica de Geração e Desaparecimento de Raios parte da premissa de que o processo de geração e desaparecimento de cada raio tem grande influência na variação do sinal recebido. De modo simplificado, pode-se ilustrar o surgimento e desaparecimento de raios na Figura 2, onde se verifica que o raio 1 surge no instante $\lg _{1}$ e desaparece no instante $\lg 1+l_{1} 1$ e, de maneira análoga, o raio 2 surge no instante $\lg 1+\lg 2$ e desaparece no instante $\lg 1+\lg 2+$ $1_{1} 2$. Os parâmetros $\lg$ e $l_{1}$ representam, respectivamente, a separação entre dois raios e o tempo médio de vida de um raio.

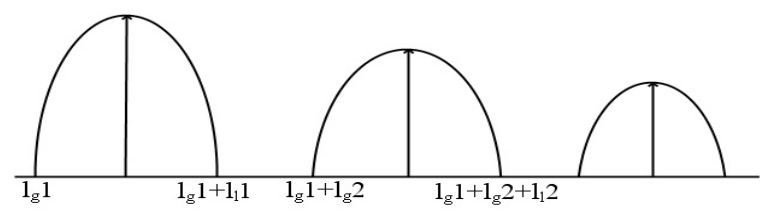

Fig. 2. Surgimento e desaparecimento de raios ao longo do tempo

A escolha da variação senoidal é para evitar mudanças abruptas na variação da amplitude dos multipercursos simulados, conforme será visto na eq. (12).

$\mathrm{O}$ número de multipercursos, $\mathrm{n}$, é determinado por um processo de Poisson, representado na eq. (2), onde $\mathrm{N}$ é o número médio de multipercursos e $p_{n}(n ; N)$ é a função densidade de probabilidade (fdp) do número de raios que chegam em um determinado instante [7]:

$$
p_{n}(n ; N)=\frac{N^{n}}{n !} \exp (-N)
$$

A separação entre dois multipercursos, $1_{\mathrm{g}}$, é considerada como tendo uma função densidade de probabilidade exponencial, $p_{g}\left(l_{g}\right)$, dada na eq. (3), cuja média é $\Delta \mathrm{L} / \mathrm{N}$, onde $\Delta \mathrm{L}$ é o tempo médio de vida de um multipercurso [7]:

$$
p_{g}\left(l_{g}\right)=\frac{N}{\Delta L} \exp \left(-\frac{N l_{g}}{\Delta L}\right)
$$

O tempo médio de vida de um raio, $l_{l}$, é representado, também, por uma função densidade de probabilidade exponencial, com média $\Delta \mathrm{L}$, ou seja [7]:

$$
p_{l}\left(l_{l}\right)=\frac{N}{\Delta L} \exp \left(-\frac{l_{l}}{\Delta L}\right)
$$

Para a modelagem do canal, o perfil de potência de retardo é suposto uma função exponencial, com um espalhamento de retardo igual a $\tau_{0}$. Assim, o retardo $\tau_{i}$, associado a cada raio, fica calculado da fdp [7]:

$$
p(\tau)=\frac{P_{R}}{\tau_{0}} \exp \left(-\frac{\tau}{\tau_{0}}\right)
$$

onde $\mathrm{P}_{\mathrm{R}}$ é a potência média recebida, calculada da seguinte equação, proposta por Rappaport [8]:

$$
P_{R}[\mathrm{dBm}]=P_{\text {cal }}[\mathrm{dBm}]+10 \log \left(\frac{A_{N R}}{A_{N c a l}}\right)
$$

$P_{c a l}$ é a potência recebida durante a calibração do sistema de medição (transmissor e receptor back-to-back), $\mathrm{A}_{\mathrm{NR}}$ é a área sob cada perfil de potência de retardos recebido durante a amostragem do canal, e $\mathrm{A}_{\mathrm{Ncal}}$ é a área sob a curva do perfil de potência de retardos medido durante a calibração.

Para a simulação do canal UWB, parâmetros do canal sondado são usados como entrada. Dentre eles, estão o espalhamento de retardo $\left(\tau_{0}\right)$, o número médio de raios $(\mathrm{N})$, o tempo médio de vida de um raio $(\Delta \mathrm{L})$, o retardo médio $\left(\tau_{\mathrm{d}}\right)$, e a potência média recebida $\mathrm{P}_{\mathrm{R}}$. De posse de cada retardo simulado, $\tau_{\mathrm{i}}$, e da potência média de cada raio, $\mathrm{P}_{\mathrm{i}}\left(\tau_{\mathrm{i}}\right)$, obtida da equação: 


$$
P_{i}(\tau)=\alpha \frac{P_{R}}{N} \exp \left(-\frac{\tau}{\tau_{p}}\right)
$$

onde $\alpha$ é um parâmetro independente de $\tau$, a ser determinado pela relação:

$$
\alpha=\frac{\tau_{p}+\tau_{d}}{\tau_{p}}
$$

e $\tau_{p}$ é o retardo quando a potência recebida se iguala a $1 / e$, aproximadamente $37 \%$, ou ainda:

$$
1 / \tau_{0}=1 / \tau_{\mathrm{d}}+1 / \tau_{\mathrm{p}}
$$

a resposta ao impulso do i-ésimo raio, $g_{i}(\tau, x)$, é obtida de:

onde:

$$
g_{i}(\tau, x)=a_{i}(x) \delta\left(\tau-\tau_{i}(x)\right)
$$

$$
a_{i}(x)=r_{i}(x) \exp \left(j \theta_{i}\right)
$$

e:

$$
r_{i}(x)=\left\{\begin{array}{c}
\sqrt{2 P_{i}} \operatorname{sen}\left(\frac{\pi}{\Delta x_{i}}\left(x-x_{i, \min }\right)\right) p / x_{i, \min } \leq x \leq x_{i, \max } \\
0 \ldots \ldots \ldots p / x<x_{i, \min } \ldots \text { ou } \ldots x>x_{i, \max }
\end{array}\right.
$$

e ainda: $x_{i, \min }$ e $x_{i, \max }$ são os pontos de geração $\left(1_{\mathrm{g}} 1+\ldots+1_{\mathrm{g}} \mathrm{i}\right)$ e desaparecimento $\left[\left(\mathrm{l}_{\mathrm{g}} 1+\ldots+\mathrm{l}_{\mathrm{g}} \mathrm{i}\right)+\mathrm{l}_{\mathrm{l}} \mathrm{i}\right]$ do i-ésimo raio; $\theta_{i}$ é a fase inicial do i-ésimo raio, distribuída uniformemente no intervalo $[0,2 \pi) ; r_{i}$ é a amplitude do i-ésimo raio e varia como uma senóide no intervalo: $x_{i, \min } \leq x \leq x_{i, \max }$, como mostrado na Figura 2.

Finalmente, o perfil de potência de retardo pode ser modelado como a resposta ao somatório de impulsos, $g(\tau, x)$, ou seja:

$$
g(\tau, x)=\sum_{i}^{\infty} g_{i}(\tau, x)
$$

onde o limite infinito é, no caso discreto, substituído pelo número de raios (n) gerados para o perfil simulado.

\section{APLICAÇÃo DO MODELO AO CANAL UWB SONDADO}

Adotando-se as fdp's exponenciais das eq. (3) a (5), as mesmas são geradas através do método computacional, denominado Método da Transformação [9], onde é preciso inverter a expressão:

obtendo-se:

$$
u=F_{x}(x)=1-e^{-\lambda x},
$$

$$
X=-\frac{1}{\lambda} \ln (1-U)
$$

onde $U$ é uniformemente distribuído no intervalo [0,1] e $\lambda$ é a média da distribuição. Nas respectivas equações citadas, $X$ passa a ser as variáveis $l_{g}, l_{l}$ e $\tau$ desejadas.
Como $U$ e 1-U são distribuídos uniformemente, a eq. (7) é simplificada e obtém-se, para as equações (3) a (5):

$$
\begin{aligned}
& p_{g}\left(l_{g}\right)=-\frac{\Delta L}{N} \ln (U) \\
& p_{l}\left(l_{l}\right)=-\Delta L \ln (U) \\
& p(\tau)=-P_{R} \tau_{0} \ln (U)
\end{aligned}
$$

O programa para a simulação do canal UWB, empregando a técnica de geração e desaparecimento de raios, foi desenvolvido em software Matlab, conforme o diagrama mostrado na Figura 3.

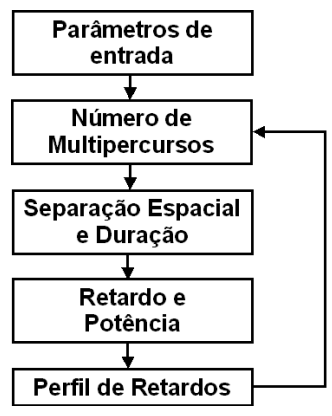

Fig. 3. Fluxo de Programação

Os parâmetros de entrada necessários para o modelo foram obtidos a partir de medições de sinal UWB, realizadas em três ambientes indoor do Bloco D da Escola de Engenharia da Universidade Federal Fluminense, denominados A, B e C, e mostrados nas Fig. 4 a 6.

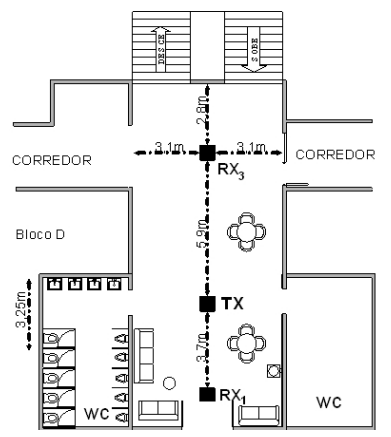

Fig. 4. Planta baixa do ambiente A

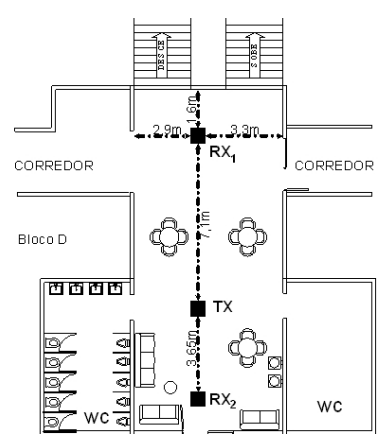

Fig. 5. Planta baixa do ambiente B 


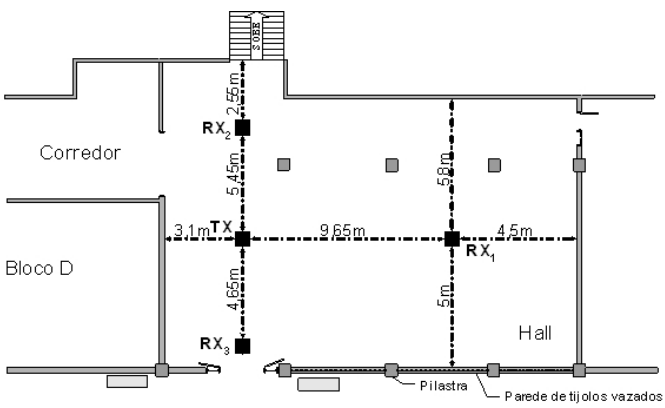

Fig. 6. Planta baixa do ambiente C

Os ambientes A e B, representados nas Fig. 4 e 5, são o $2^{\circ}$ e $3^{\circ}$ andar, respectivamente. É importante observar que o ambiente B é muito semelhante ao ambiente A, mas foram utilizadas posições diferentes para o transmissor e o receptor. O ambiente $\mathrm{C}$, na Fig.6, é o hall de entrada do prédio de Engenharia, mais amplo que os ambientes A e B. Para cada ambiente, o transmissor (TX) foi colocado fixo, inicialmente, enquanto o receptor (RX) foi deslocado. Em seguida, RX foi mantido fixo e TX foi movimentado.

Uma média dos parâmetros espalhamento de retardo, número médio de multipercursos, tempo médio de vida de um raio, retardo médio e potência média recebida, foi determinada para cada ambiente, a fim de ser aplicada como parâmetro de entrada da simulação.

A tabela I apresenta os valores configurados para a campanha de medidas [10], onde a técnica de varredura de freqüência foi empregada para a sondagem do canal UWB.

Tabela I

Dados da Sondagem no Canal

Dados da Sondagem no Canal
\begin{tabular}{|l|c|c|}
\hline Parâmetros & Valor & Unidade \\
\hline Faixa de Freqüência & 960 a 1710 & $\mathrm{MHz}$ \\
\hline Largura de Banda & 750 & $\mathrm{MHz}$ \\
\hline $\begin{array}{l}\text { Espaçamento, em Freqüência, entre } \\
\text { Amostras - } \Delta \mathrm{f}\end{array}$ & 0,46875 & $\mathrm{MHz}$ \\
\hline Número de Amostras Varridas & 1601 & - \\
\hline Resolução Temporal $-\Delta \tau$ & 1,33 & $\mathrm{~ns}$ \\
\hline Retardo Máximo $-\tau_{\mathrm{MAX}}$ & 2133 & $\mathrm{~ns}$ \\
\hline Tempo de varredura & 696 & $\mathrm{~ms}$ \\
\hline
\end{tabular}

Como produtos da sondagem do canal, os parâmetros de pequena escala, retardo médio e espalhamento de retardo, de cada ambiente de medição (A, B e C) foram determinados e os valores médios para cada ambiente de medidas estão relacionados na Tabela II.

Tabela II

\begin{tabular}{|l|c|c|c|}
\hline \multirow{2}{*}{ Resultados da Sondagem no Canal } \\
\cline { 2 - 4 } \multicolumn{2}{|c|}{ Parâmetros } & \multicolumn{2}{c|}{ Ambiente de Medidas } \\
\cline { 2 - 4 } & A & B & C \\
\hline Espalhamento de retardo (ns) & 12,97 & 14,16 & 20,86 \\
\hline Retardo Médio (ns) & 22,14 & 23,04 & 29,76 \\
\hline Número de multipercursos & 30 & 35 & 37 \\
\hline
\end{tabular}

A Fig. 7 mostra um exemplo de simulação utilizando a Técnica de Geração e Desaparecimento de Raios, gerando 500 perfis de retardo, ao longo do tempo.

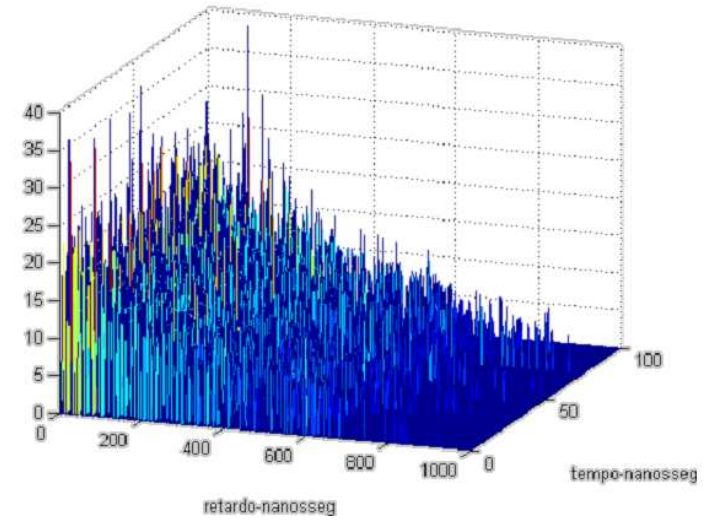

Fig. 7. Exemplo de simulação, com 500 perfis gerados

\section{RESUltados DAS SimulaçõES}

Para cada ambiente sondado, foram simulados 500 perfis de potência de retardo e a distribuição de probabilidade do espalhamento de retardos simulado foi comparada à distribuição do espalhamento obtido a partir dos dados processados, relativos aos ambientes medidos. Nos gráficos das fig. 8,9 e 10 , tais distribuições são traçadas com linha pontilhada, para a simulada, e com linha cheia, para a medida. Tais figuras são relativas aos ambientes A, B e C, respectivamente. Analisando as Funções Distribuição é possível verificar que em todos os ambientes a simulação contempla a faixa de valores medidos, havendo maior discrepância no ambiente C, exatamente o ambiente de maiores dimensões.

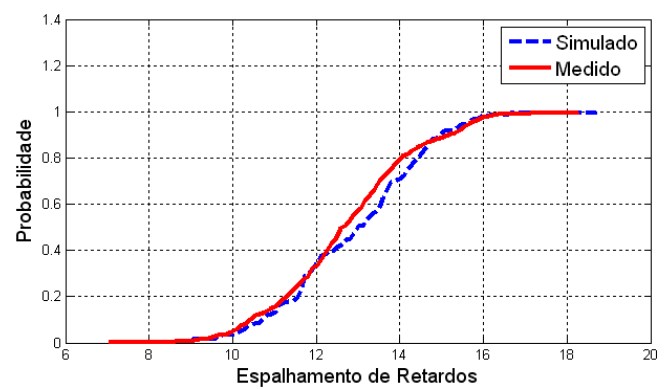

Fig. 8. Função distribuição de probabilidade do espalhamento de retardo no ambiente $\mathrm{A}$

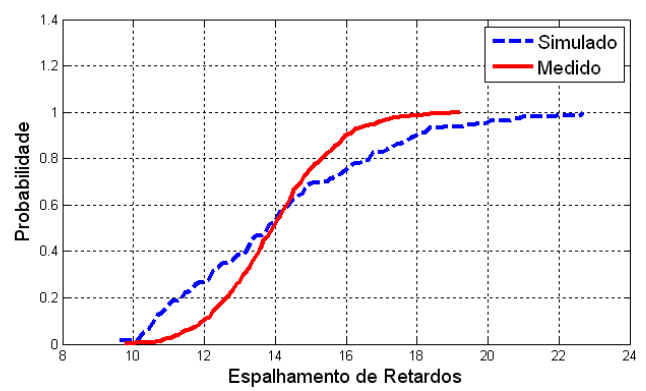

Fig. 9. Função distribuição de probabilidade do espalhamento de retardo no ambiente B 


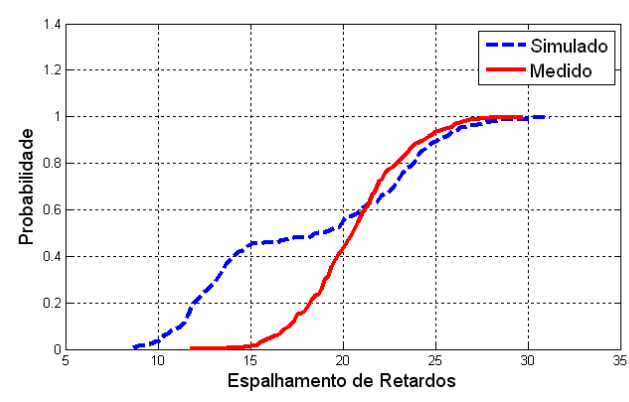

Fig. 10. Função distribuição de probabilidade do espalhamento de retardo no ambiente $\mathrm{C}$

Nos gráficos das Figuras 11 a 13, relacionando o espalhamento de retardo versus o número de perfis de retardo simulados, verifica-se que, com a Técnica de Geração e Desaparecimento de Raios, tem-se uma convergência no entorno de $99 \%$ entre o valor medido e simulado para todos os ambientes sondados, a partir de 400 perfis simulados.

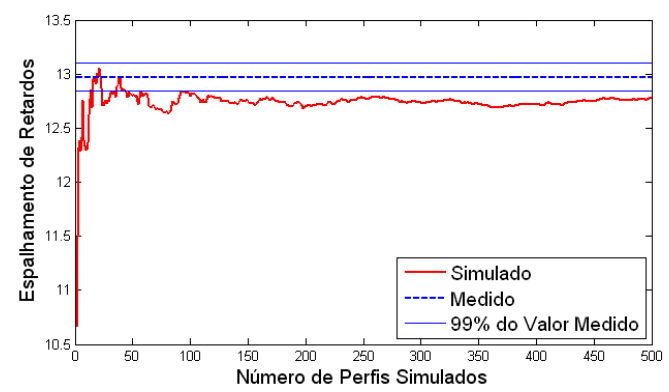

Fig.11. Espalhamento de retardo x Número perfis para o ambiente A

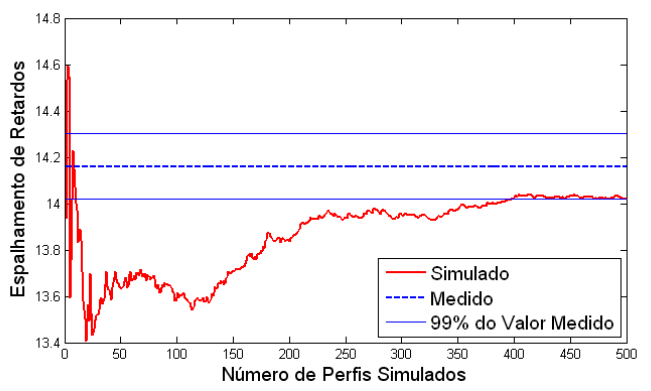

Fig. 12. Espalhamento de retardo x Número perfis para o ambiente B

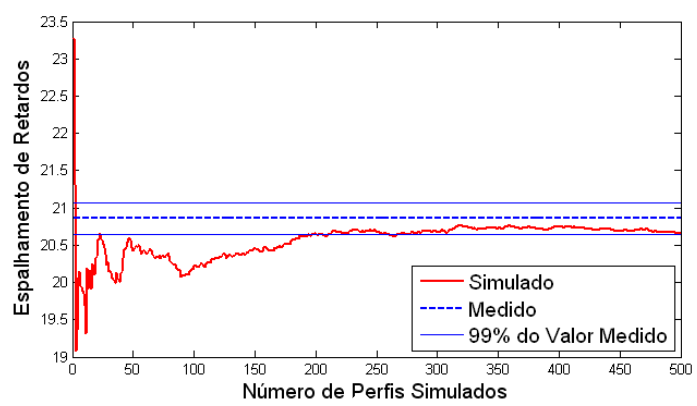

Fig. 13. Espalhamento de retardo x Número perfis para o ambiente $\mathrm{C}$

\section{CONCLUSÕES}

A tecnologia UWB cobre, com altas taxas, ambientes indoor, já que trabalha com faixa ultra-larga de frequência e baixa relação sinal-ruído. É, portanto, atrativa para aplicação indoor, nos dias de hoje, quando altas taxas de transmissão estão envolvidas.

Este artigo abordou a simulação de canal UWB indoor, numa faixa de 760 - $1910 \mathrm{MHz}$, empregando uma técnica de modelagem desenvolvida para canal CDMA, denominada de Técnica de Geração e Desaparecimento de Raios. Utilizando os dados da sondagem realizada em três ambientes indoor da Escola de Engenharia da UFF, foi demonstrado que a técnica funciona adequadamente para o canal UWB de $750 \mathrm{MHz}$, já que os valores medidos se encontram dentro da faixa de valores simulados. No ambiente indoor mais amplo observa-se maior afastamento entre os valores menores de espalhamento de retardo simulado e medido, havendo boa concordância nos valores acima da média. Ocorre convergência relativamente rápida para os valores de espalhamento de retardo simulados e, com 400 simulações, já se atinge cerca de $99 \%$ de convergência. Destaca-se, ainda, que uma das grandes vantagens da utilização desta técnica é que tem uma aplicação relativamente mais simples que outros modelos físicos, entre eles, o desenvolvido por Hashemi.

$\mathrm{Na}$ continuação deste trabalho serão analisados outros parâmetros para validação da técnica, entre eles retardo médio e banda de coerência. Deverão ser realizadas mais medições nos ambientes analisados com a finalidade de melhorar a modelagem do canal UWB indoor. Também serão analisados ambientes outdoor para pequenas distâncias e indoor-outdoor, de forma a verificar a validade do modelo para os mesmos.

\section{REFERÊNCIAS}

[1] C. F. Souza e J. C. R. Dal Bello, UWB Signals Transmission in Outdoor Environments for Emergency Communications, WCEMP2008

[2] J. D. Parsons, The Mobile Radio Propagation Channel, New York: John Wiley \& Sons, 1992.

[3] A. G. Guimarães, Modelagem e Simulação de Canais Móveis, Tese de Mestrado, IME, jan 1998.

[4] G. L. Turin, F. D. Clapp, T. L. Johnston, S. B. Fine, D. Lavry, A Statistical Model of Urban Multipath Propagation, IEEE Trans. on Vehic. Techn, Vol. VT-21, fev 1972.

[5] H. Suzuki, A Statistical Model for Urban Radio Propagation, IEEE Trans. on Comumm., vol. COM-25, jul 1977.

[6] H. Hashemi, Simulation of the Urban Radio Propagation Channel, IEEE Trans. on Vehic. Techn., Vol VT-28, pp 213-225, aug 1979.

[7] H. Iwai, Y. Karasawa, Wideband Propagation Model for the Analysis of the Effect of the Multipath Fading on the Near-Far Problem in CDMA Mobile Radio Systems, IEICE Trans. Comm., Vol E 76-B, pp-103-112, fev 1993.

[8] W. G. Newhall, K. Saldanha, T. S. Rappaport, Using RF Channel Sounding Measurements to Determine Delay Spread and Path Loss, jan 1996.

[9] A. Leon-Garcia, Probability and Random Processes for Eletrical Enginiiring, jul 1993.

[10] C. F. Souza, Análise da Dispersão Temporal de Canais de Banda Ultralarga (UWB) através de Medidas Realizadas em Ambientes Internos e Externos, Tese de Mestrado, UFF, 2006. 